\title{
Urinary microbiota in patients with prostate cancer and benign prostatic hyperplasia
}

Haining $\mathrm{Yu}^{1}$, Hongzhou Meng ${ }^{2}$, Feng Zhou ${ }^{2}$, Xiaofeng Ni ${ }^{3}$, Shengrong Shen ${ }^{3}$, Undurti N. Das ${ }^{4}$

${ }^{1}$ College of Pharmaceutical Sciences, Zhejiang University of Technology, Hangzhou, China

2Department of Urology, The First Affiliated Hospital, Zhejiang University, Hangzhou, China

${ }^{3}$ Department of Food Science and Nutrition, Zhejiang University, Hangzhou, China ${ }^{4}$ UND Life Sciences, USA

Submitted: 6 May 2014

Accepted: 12 June 2014

Arch Med Sci 2015; 11, 2: 385-394

DOI: $10.5114 /$ aoms.2015.50970

Copyright @ 2015 Termedia \& Banach

\section{Abstract}

Introduction: Inflammation is associated with promotion of the initiation of various malignancies, partly due to bacterial infection-induced microenvironmental changes. However, the exact association between microbiota in urine, seminal fluid and the expressed prostatic secretions and benign prostatic hypertrophy and prostate cancer is not clear.

Material and methods: In the present study, we investigated the type of microbiota in the expressed prostatic secretions (EPS) of patients with prostate cancer and benign prostatic hyperplasia (BPH) by the polymerase chain reaction-denaturing gradient gel electrophoresis (PCR-DGGE) method using universal bacterial primers. In order to understand the possible association between various bacteria and prostate cancer, quantitative real-time PCR assay was performed to quantify the amount of strains of bacteria in urine, EPS and seminal fluid.

Results: The prostate cancer group had a significantly increased number of Bacteroidetes bacteria, Alphaproteobacteria, Firmicutes bacteria, Lachnospiraceae, Propionicimonas, Sphingomonas, and Ochrobactrum, and a decrease in Eubacterium and Defluviicoccus compared to the BPH group. The number of Escherichia coli in the prostate cancer group was significantly decreased in urine and increased in the EPS and seminal fluid, while the number of Enterococcus was significantly increased in the seminal fluid with little change in urine and EPS.

Conclusions: Based on these results, we suggest that there are significant changes in the microbial population in EPS, urine and seminal fluid of subjects with prostate cancer and BPH, indicating a possible role for these bacteria in these two conditions.

Key words: urinary microbiota structure, prostate cancer, polymerase chain reaction-denaturing gradient gel electrophoresis, quantitative real-time polymerase chain reaction.

\section{Introduction}

Prostate cancer is one of the most common cancers in men, especially in the elderly. Morbidity due to prostate adenocarcinoma increases to $\sim 60 \%$ for men over the age of 60 years in the USA. It has long been suspected that chronic inflammation may have an important role in the initiation and progression of prostate cancer [1-3]. The observation that

\author{
Corresponding authors: \\ Dr. Shengrong Shen PhD \\ Department of Food \\ Science and Nutrition \\ School of Biosystems \\ Engineering and Food \\ Science \\ Zhejiang University \\ Hangzhou 310058, China \\ Phone: 8657188982539 \\ E-mail: shrshen@zju.edu.cn \\ Dr. Undurti N. Das MD, FAMS, \\ FRSC \\ UND Life Sciences \\ 2020 S $360^{\text {th }}$ St \\ K-202, Federal Way \\ WA 98003, USA \\ E-mail: undurti@hotmail.com
}


Helicobacter pylori induces chronic inflammation and thus may pave the way to develop stomach cancer [3] lends support to such a belief that bacteria may also have a role in prostate cancer [3]. There is some support to this contention that prostatic inflammation may increase the risk of benign prostatic hyperplasia $(\mathrm{BPH})$ and prostate cancer $[4,5]$.

It is difficult to detect many anaerobic bacteria present in various human body fluids, especially in the urine, expressed prostatic secretions (EPS) and seminal fluid, using traditional culture methods $[6,7]$. Hence, in the present study, we employed culture-independent methods (polymerase chain reaction-denaturing gradient gel electrophoresis - PCR-DGGE) that are more reliable to detect various bacteria present in the human body fluids [8]. This method has previously been used by others to identify a variety of bacteria in various secretion of the human body [8-12].

Gram-negative enteric bacteria including Escherichia coli, Pseudomonas aeruginosa, and Enterococcus are frequently associated with urinary tract infections that may cause chronic bacterial prostatitis [13]. Furthermore, infections due to E. coli and Enterococcus are accompanied by elevated levels of pro-inflammatory cytokines that are related to cancer development and progression [14, 15]. Hence, it is reasonable to propose that chronic bacterial prostatitis induced by bacteria may enhance proinflammatory responses that may contribute to prostate cancer.

In order to verify this possibility, we investigated the bacterial population in the EPS of patients with BPH and prostate cancer by PCR-DGGE with $16 S$ rDNA methods and quantified changes in the number of E. coli and Enterococcus by quantitative real-time PCR assay in the present study $[16,17]$.

\section{Material and methods}

\section{Specimen collection}

All male patients included in the study were under the age of 75 and diagnosed with BPH or prostate cancer. All patients were educated as to how to collect their urinary samples without contamination. The penis was cleaned with warm water and a 75\%-alcohol tampon 3 times [18] and urine was collected in a 10-ml sterile tube by each study subject. Expressed prostatic secretions and seminal fluid were collected following prostatic massage in a $5.0-\mathrm{ml}$ sterile tube for each subject. Urine, EPS and seminal fluid were carefully collected into the tubes without touching the interior wall of the sterile tube. Routine urine test was performed before sample storage. Urine, EPS and seminal fluid were all stored at $-80^{\circ} \mathrm{C}$ for DNA extraction. The EPS samples from prostate cancer/BPH patients were pooled for the DGGE analysis.

\section{DNA extraction}

Samples were melted to liquid at room temperature $\left(15-25^{\circ} \mathrm{C}\right)$ and stored on ice for DNA extraction. QIAamp DNA Mini Kit (Qiagen, USA) and distilled water were equilibrated to room temperature before DNA extraction. Twenty $\mu \mathrm{l}$ QIAGEN Protease was pipetted into the bottom of a $1.5 \mathrm{ml}$ micro-centrifuge tube, and then a $200 \mu \mathrm{l}$ sample (urine, EPS and seminal fluid) was added to the tube. Following this, $200 \mu \mathrm{l}$ of Lysis buffer was added to the sample and mixed for $15 \mathrm{~s}$, and the mixture was incubated at $56^{\circ} \mathrm{C}$ for $10 \mathrm{~min}$ in a water bath. Two hundred $\mu$ of absolute ethanol was pipetted into the mixture and mixed well. Following brief centrifugation, the mixture was carefully applied to a QIAamp Mini spin column without wetting the rim. On the other hand, following 1-min-long centrifugation at $8000 \mathrm{rpm}$, $50 \mu \mathrm{l}$ of buffer AW1 was carefully added to the column without wetting the rim. An additional 1-minlong centrifugation at $8000 \mathrm{rpm}$ was performed and the column was placed in a clean $2 \mathrm{ml}$ collection tube. Five hundred $\mu$ l buffer AW2 was added to the column and was centrifuged for $3 \mathrm{~min}$ at $14,000 \mathrm{rpm}$. Subsequently the column was kept in a clean $1.5 \mathrm{ml}$ tube, $20 \mu \mathrm{l}$ Elution buffer was added, and it was incubated at room temperature for $1 \mathrm{~min}$. DNA was collected from the columns by centrifuging at $8000 \mathrm{rpm}$ for $1 \mathrm{~min}$. The DNA samples collected were subpackaged and stored at $-20^{\circ} \mathrm{C}$.

\section{Polymerase chain reaction amplification}

The concentrations of DNA in the samples were measured by ultraviolet spectrophotometer (Thermo Electron Corporation, USA). All DNA samples were amplified in the $\mathrm{V} 3$ regions of the $16 \mathrm{~S}$ rRNA using universal bacterial primers (341F:5'-GTATTACCGCGGCTGCTGG-3'; 534R:5'-ACTCCTACGGGAGGCAGCAG-3') with a 40-bp GC clamp (5'-CGCCCGCCGCGCGCGGCGGGCGGGGCGGGGGCACGGGGGG-3') attached to the 5' ends of the reserve primers (534R) for DGGE analysis. The PCR mixture comprised $1 \mu \mathrm{l}$ of Bestar Taq DNA Polymerase (2.5 U/ $\mu \mathrm{l}$, DBI Bioscience, Shanghai, China), $5 \mu \mathrm{l}$ of deoxynucleoside triphosphates (dNTPs, $2 \mathrm{mM}$ each), $5 \mu$ l of $10 \times$ Bestar Taq Buffer, $1 \mu$ l of each primer $(10 \mu \mathrm{M})$ and $2 \mu$ l of extracted bacterial DNA $(\sim 60 \mathrm{ng})$. The total PCR reaction volume was $50 \mu \mathrm{l}$. The protocol for the PCR procedure was the touchdown protocol as described by Muyzer et al. [8] as follows: $94^{\circ} \mathrm{C}$ for $5 \mathrm{~min}$; 20 cycles of touchdown PCR: denaturation at $95^{\circ} \mathrm{C}$ for $30 \mathrm{~s}$, annealing at $65^{\circ} \mathrm{C}$ for $30 \mathrm{~s}$ with the temperature decreasing by 
$0.5^{\circ} \mathrm{C} /$ cycle until the annealing temperature was $55^{\circ} \mathrm{C}$, and extension at $72^{\circ} \mathrm{C}$ for $30 \mathrm{~s} ; 20$ cycles of PCR: $95^{\circ} \mathrm{C}$ for $30 \mathrm{~s}, 55^{\circ} \mathrm{C}$ for $30 \mathrm{~s}$, and $72^{\circ} \mathrm{C}$ for $30 \mathrm{~s}$; final extension at $72^{\circ} \mathrm{C}$ for $5 \mathrm{~min} ; 4^{\circ} \mathrm{C}$ hold. Polymerase chain reaction (PCR) was performed in a thermal cycler PCR system (BBI, Canada) and PCR products were checked by electrophoresis on $1.0 \%(\mathrm{w} / \mathrm{v})$ agarose gel (Biowest Regular Agarose G-10, Spain). Electrophoresis was performed at a voltage of $100 \mathrm{~V}$ for 20 min with $1 \times$ TAE buffer, and visualized by ethidium bromide staining using a gel imaging system (Bio-Rad Laboratories GelDoc 2000, Oslo, Norway). All PCR products were stored at $-20^{\circ} \mathrm{C}$ before DGGE electrophoresis.

\section{Denaturing gradient gel electrophoresis}

Denaturing gradient gel electrophoresis analysis was performed for the EPS specimens of prostate cancer and $\mathrm{BPH}$, using the Bio-Rad Dcode mutation detection system (Bio-Rad, Hercules, CA, USA) according to the manufacturer's instructions. DNA fragments with different sequences were separated in $8 \%$ polyacrylamide (acrylamide: bisacrylamide $=37.5: 1$; w/v) gels in $1 \times$ TAE buffer with $200 \mathrm{ng}$ of each PCR product. A $30-60 \%$ denaturant gradient (100\% denaturant was $7 \mathrm{~mol} / \mathrm{l}$ urea and $40 \%$ (v/v) deionized formamide) was applied in the DGGE electrophoresis, which was performed in $1 \times \mathrm{TAE}$ buffer at $180 \mathrm{~V}$ at $60^{\circ} \mathrm{C}$ for $4 \mathrm{~h}$. Subsequently, the gels were washed with ultrapure water and stained with $5 \%$ Goldview dye for $30 \mathrm{~min}$ and photographed. DGGE graphs were digitized by Quantity One Analysis software (Gene Genius).

\section{Denaturing gradient gel electrophoresis} band sequencing and phylogenetic analysis

Denaturing gradient gel electrophoresis bands (\#5, \#6, \#8, \#10, \#13, \#15, \#16, \#17, \#19) excised from the gel under UV and the DNA fragments were collected and purified with DNA Gel Extraction Kit (SK1135, Sangon, Shanghai, China) according to the manufacturer's instructions. The purified DNA was used as templates for PCR with the primers mentioned above. The PCR products were ligated into the PUCm-T vector (Sangon, Shanghai, China) and competent E. coli $\mathrm{DH} 5 \alpha$ cells (Sangon) were transformed. The recombinant cells were selected and cultured overnight at $37^{\circ} \mathrm{C}$. Subsequently, bacteria were collected, and DNA with the plasmid was extracted using the UNIQ-10 column kit (Sangon) following the kit instructions. Clones located in the same position as the original DGGE bands were sequenced (Sangon, Shanghai, China).

Similarity searches with obtained sequences were performed online based on the NCBI GenBank database (http://www.ncbi.nlm.nih.gov) BLAST. We compared the 16S rDNA sequences of the selected bands and their identity was arrived at (by comparing with the NCBI GenBank database) when the similarity of the sequence was above $97 \%$. Based on the similarity search results, a phylogenetic tree was constructed by the neighbor-joining method with the MEGA 5 software (version 5.05). The consistency of the relationship on the tree was assessed by bootstrap resampling. Bootstrapping was performed for 1000 trials in accordance with the Clustal X program [19].

\section{qPCR assay for Escherichia coli and Enterococcus}

Each DNA sample in this study was amplified with primers (VS8F: 5'-GGCGGATTAGACTTCGGCTA-3', VS9R:5'-CGTTTTGGCACTATTTGCCC-3') for E. coli and primers (Ent1F: 5'-TACTGACAAACCATTCATGATG-3', Ent2R:5'-AACTTCGTCACCAACGCGAAC-3') for Enterococcus. Each PCR reaction mix consisted of the following in a total volume of $20 \mu \mathrm{l}: 10 \mu \mathrm{l}$ of Bestar SybrGreen qPCR mastermix (DBI Bioscience, Shanghai, China), $0.5 \mu$ l of each PCR primer $(10 \mu \mathrm{M}), 2.5 \mu \mathrm{l}$ of DNA template and $6.5 \mu \mathrm{l}$ of distilled water. Polymerase chain reaction conditions employed were as follows: initial denaturation at $95^{\circ} \mathrm{C}$ for $2 \mathrm{~min} ; 40$ cycles for PCR reaction of $95^{\circ} \mathrm{C}$ for $10 \mathrm{~s}, 55^{\circ} \mathrm{C}$ for $30 \mathrm{~s}$ and $72^{\circ} \mathrm{C}$ for $30 \mathrm{~s}$; melting curve at $65^{\circ} \mathrm{C}$ for $5 \mathrm{~s}$ and 60 cycles from $65^{\circ} \mathrm{C}$ to $95^{\circ} \mathrm{C}$ (10 s/cycle, $0.5^{\circ} \mathrm{C} /$ cycle).

A DNA sample with the maximum concentration was diluted $10,10^{2}, 10^{3}, 10^{4}, 10^{5}$ times and amplified under the same PCR conditions for the standard curves. All the samples were amplified in triplicate using Thermal Cycler (CFX-Touch, BIO RAD) and the Ct values were compared with the standard curve to obtain the relative quantity of E. coli and Enterococcus.

\section{Statistical analysis}

All results obtained were expressed in mean \pm SD. Statistical analysis was performed by analysis of variance or by paired $t$-test when just two values were compared, using SPSS software version 9.1. Group differences were shown as * meaning $p<0.05$, ${ }^{* *}$ meaning $p<0.01$, or ${ }^{* * *}$ meaning $p<0.001$.

\section{Results}

\section{Specimen characteristics}

Specimens for the present study were obtained from patients with $\mathrm{BPH}$ and prostate cancer attending the Department of Urology, the First Affiliated Hospital of the College of Medicine, Zhejiang University. The mean age of the recruited subjects in both groups was 66.7 years. Urine and blood 
Table I. Urine characteristics of patients with $\mathrm{BPH}$

\begin{tabular}{|c|c|c|c|c|}
\hline Patient no. & Occult blood & WBC $[/ \mu \mathrm{ll}]$ & Turbidity & Bacteria $[/ \mu \mathrm{l}]$ \\
\hline 14 & - & 9.6 & + & 2.6 \\
\hline 15 & - & 7.4 & - & 22.3 \\
\hline 16 & - & 4.6 & - & 32.2 \\
\hline 17 & _ & 11.9 & _ & 11.9 \\
\hline 18 & + & 23.1 & _ & 9.4 \\
\hline 19 & ++ & 8.5 & - & 7.1 \\
\hline 20 & $+++($ OVER $)$ & 21.1 & - & 5.5 \\
\hline 21 & $+++($ OVER $)$ & 80.0 & - & 7.1 \\
\hline 22 & _ & 2.3 & _ & 10.4 \\
\hline 23 & + & 18.5 & _ & 70.2 \\
\hline 24 & + & 21.5 & - & 87.3 \\
\hline 25 & ++ & 207.7 & + & 16.7 \\
\hline 26 & + & 2.0 & _- & 7.9 \\
\hline 27 & ++ & 101.8 & + & 12.7 \\
\hline 28 & - & 2.2 & - & 8.7 \\
\hline 29 & $+++($ OVER $)$ & 34.5 & - & 3.1 \\
\hline 30 & ++ & 18.2 & _ & 1.8 \\
\hline 31 & ++ & 14.4 & _- & 7.9 \\
\hline 32 & + & 16.3 & _- & 6.6 \\
\hline 33 & + & 5.6 & - & 0.7 \\
\hline 34 & ++ & 52.3 & 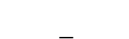 & 11.9 \\
\hline
\end{tabular}

WBC - white blood cells.

Table II. Urine characteristics of patients with prostate cancer

\begin{tabular}{|c|c|c|c|c|}
\hline Patient no. & Occult blood & $\mathrm{WBC}[/ \mu \mathrm{l}]$ & Turbidity & Bacteria $[/ \mu \mathrm{l}]$ \\
\hline 1 & $+++($ OVER) & 444.9 & + & 22.3 \\
\hline 2 & $+++($ OVER $)$ & 131.1 & + & 68.3 \\
\hline 3 & $+++($ OVER $)$ & 157.4 & - & 5291.8 \\
\hline 4 & ++ & 431.9 & + & 23.0 \\
\hline 5 & ++ & 331.0 & + & 36.8 \\
\hline 6 & $+++(O V E R)$ & 100.6 & - & 23.0 \\
\hline 7 & $+++($ OVER $)$ & 39.2 & - & 46.7 \\
\hline 8 & $+++($ OVER $)$ & 148.5 & - & 67.1 \\
\hline 9 & ++ & 49.9 & - & 28.7 \\
\hline 10 & $+++($ OVER $)$ & 34.3 & + & 151.9 \\
\hline 11 & + & 21.5 & - & 87.3 \\
\hline 12 & - & 556.4 & + & 24602.5 \\
\hline 13 & $+++($ OVER) & 30.9 & _- & 39.1 \\
\hline
\end{tabular}


Table III. Tumor markers of patients with prostate cancer

\begin{tabular}{|lccccc|}
\hline Patient no. & $\begin{array}{c}\alpha \text {-Fetoprotein } \\
{[\mathrm{ng} / \mathrm{ml}]}\end{array}$ & CEA [ng/ml] & CA125 [U/ml] & Ferritin $[\mathrm{ng} / \mathrm{ml}]$ & PSA [ng/ml] \\
\hline 1 & 1.8 & 1.6 & 7.8 & 146.0 & 0.624 \\
\hline 2 & 3.8 & 2.4 & 19.4 & 441.0 & 5.447 \\
\hline 3 & 6.1 & 5.4 & 6.8 & 59.4 & 12.117 \\
\hline 4 & 2.2 & 1.2 & 20.9 & 6389.0 & $>1000$ \\
\hline 5 & 1.6 & 2.3 & 212.2 & 457.4 & 140.866 \\
\hline 6 & 5.1 & 4.9 & 26.9 & 399.7 & 37.694 \\
\hline 7 & 3.1 & 4.8 & 15.1 & 382.8 & 17.790 \\
\hline 8 & 2.1 & 1.4 & 6.6 & 221.7 & 28.892 \\
\hline 9 & 3.3 & 4.1 & 12.5 & 308.2 & 16.599 \\
\hline 10 & 3.7 & 4.7 & 9.8 & 171.5 & 2.936 \\
\hline 11 & 2.3 & 2.6 & 8.3 & 153.9 & 6.342 \\
\hline 12 & 2.7 & 3.2 & 4.2 & 156.3 & 11.900 \\
\hline 13 & 2.9 & 4.3 & 8.9 & 151.3 & 1.917 \\
\hline
\end{tabular}

CEA - carcino-embryonic, CA125 - carbohydrate antigen 125 .

analysis was performed before specimen collection. Characteristics of the urine examination of the recruited patients are given Tables I and II. The characteristics of the tumor markers of patients with prostate cancer studied and their results are given in Table III. Compared to patients with prostatic hyperplasia, patients with prostate cancer had higher incidence of occult blood and turbidity, and a higher quantity of white blood count (WBC) and bacteria in the urine, suggesting that these patients might be having concurrent infection and inflammation that could be due to the presence of infection of the prostate or urethritis. Three inferences could be arrived at from these findings. They are: (i) active infection is present in the prostate at the time of the study; (ii) active infection is present in the urinary tract; and (iii) active infection is present in both the prostate and the urinary tract. Despite these possibilities and possible presence of active infection in the prostate and/or urinary tract, the results of the present study showed that there could occur a close relationship between infections and prostate cancer.

\section{Polymerase chain reaction efficiency of 16s RNA}

The total DNA of each specimen was extracted successfully and PCR assays for 16sRNA gene fragments of bacteria were performed using bacterial universal primers $341 \mathrm{~F}$ and $534 \mathrm{R}$. The amplified fragments of PCR were approximately $230 \mathrm{bp}$, as shown in Figures 1 and 2. Based on the results obtained, it is evident that 16s RNA gene fragments of bacteria in the specimens had good amplification efficiency.

\section{Denaturing gradient gel electrophoresis analysis and sequencing results}

Polymerase chain reaction-denaturing gradient gel electrophoresis fingerprinting analysis of patients with $\mathrm{BPH}$ and prostate cancer for predominant bacteria was used to identify EPS microbiota

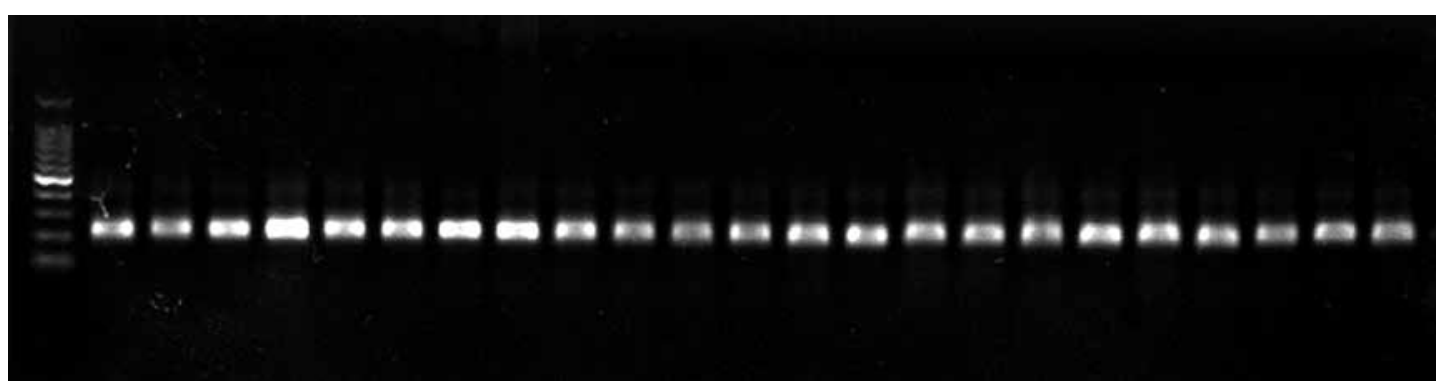

Figure 1. Gel profiles of the V3 region amplified by PCR from the extracted DNA from subjects with prostate cancer 


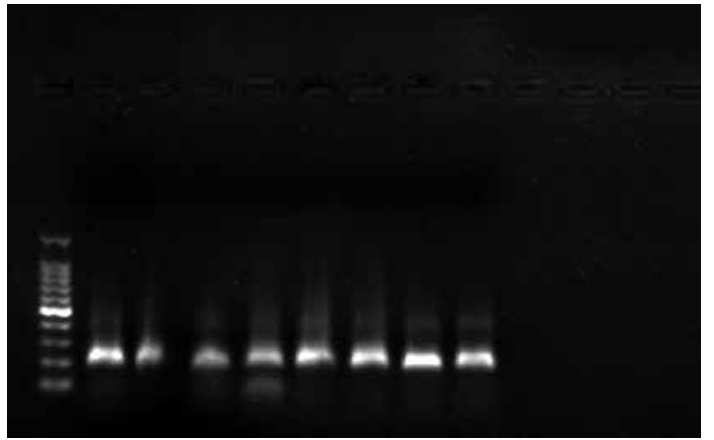

Figure 2. Gel profiles of the $\mathrm{V} 3$ region amplified by $\mathrm{PCR}$ from the extracted DNA from subjects with $\mathrm{BPH}$

(Figure 3). The extracted DNA was amplified with universal primers targeting the hypervariable $\mathrm{V} 3$ region of the $16 \mathrm{~S}$ rRNA gene. Based on PCR-DGGE fingerprinting analysis, the diversity of microbiota structures was presented with separated bands of different DNA sequences, as shown in Figure 3, which showed that there were about 20 discernible bands.

According to the DGGE profile of EPS, several bands of the prostate cancer group were discovered to be distinct from those present in the $\mathrm{BPH}$ group. These distinct bands, namely \#5, \#6, \#8, $\# 10$, \#13, \#15, \#16, \#17 and \#19, were identified as significant variables in discriminating the prostate cancer group from the BPH group. These distinct bands were sequenced to determine the exact microorganisms that were present in the urinary system during prostate cancer. The bands (\#5, \#6, \#8, \#13, \#16, \#17, and \#19) that were brighter in the prostate cancer and bands (\#10 and \#15) in the BPH groups were amplified, and the lengths of these fragments were about $190 \mathrm{bp}$ (Figure 4).

The sequences of prominent bands \#5, \#6, \#8, $\# 13$, \#16, \#17, and \#19 in the prostate cancer group matched with Bacteroidetes bacteria, Alphaproteobacteria, Firmicutes bacteria, Lachnospiraceae, Propionicimonas, Sphingomonas, Ochrobactrum and bands \#10 and \#15 of BPH matched with Eubacterium and Defluviicoccus species respectively. It is evident from these results that there were almost no significant differences in the diversity of bacteria between the groups except for the fact that seven kinds of microbes in the prostate cancer group were distinctly increased in quantity compared with those in the $\mathrm{BPH}$ group. However, Eubacterium and Defluviicoccus were present in lower amounts in the prostate cancer group compared with the $\mathrm{BPH}$ group.

\section{Phylogenetic analysis}

Sequences of the bands were blasted in the NCBI database and a sequence having above $97 \%$ similarity was regarded as the same. Every

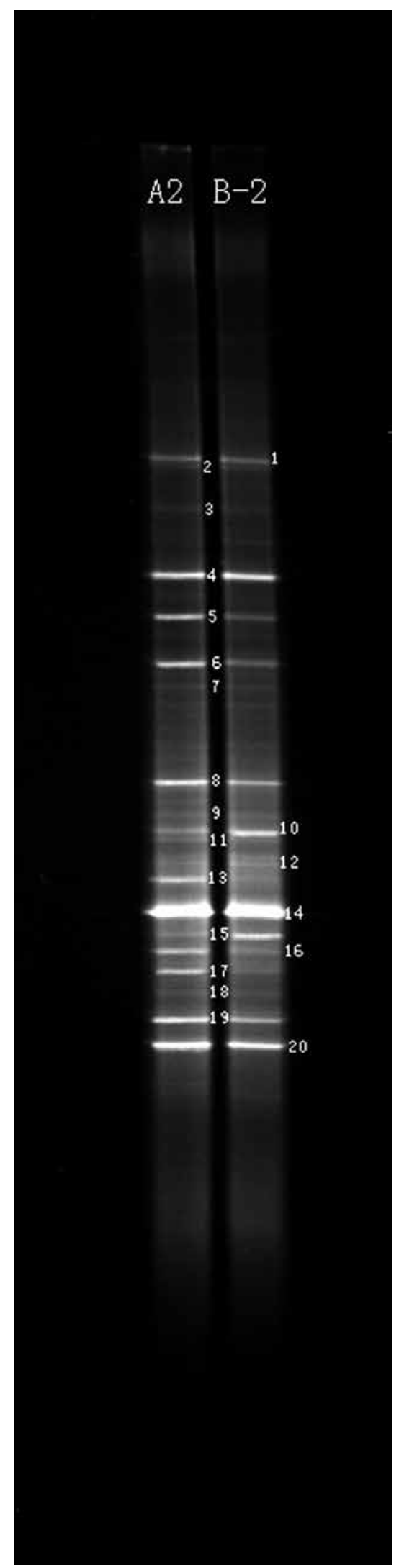

Figure 3. Gel profiles of the $\mathrm{V} 3$ region amplified by polymerase chain reaction from bands \#5, \#6, \#8, $\# 10, \# 13$, \#15, \#16, \#17 and \#19. The lengths of fragments were approximately $190 \mathrm{bp}$ (BPH (A2) and prostate cancer (B-2)) 


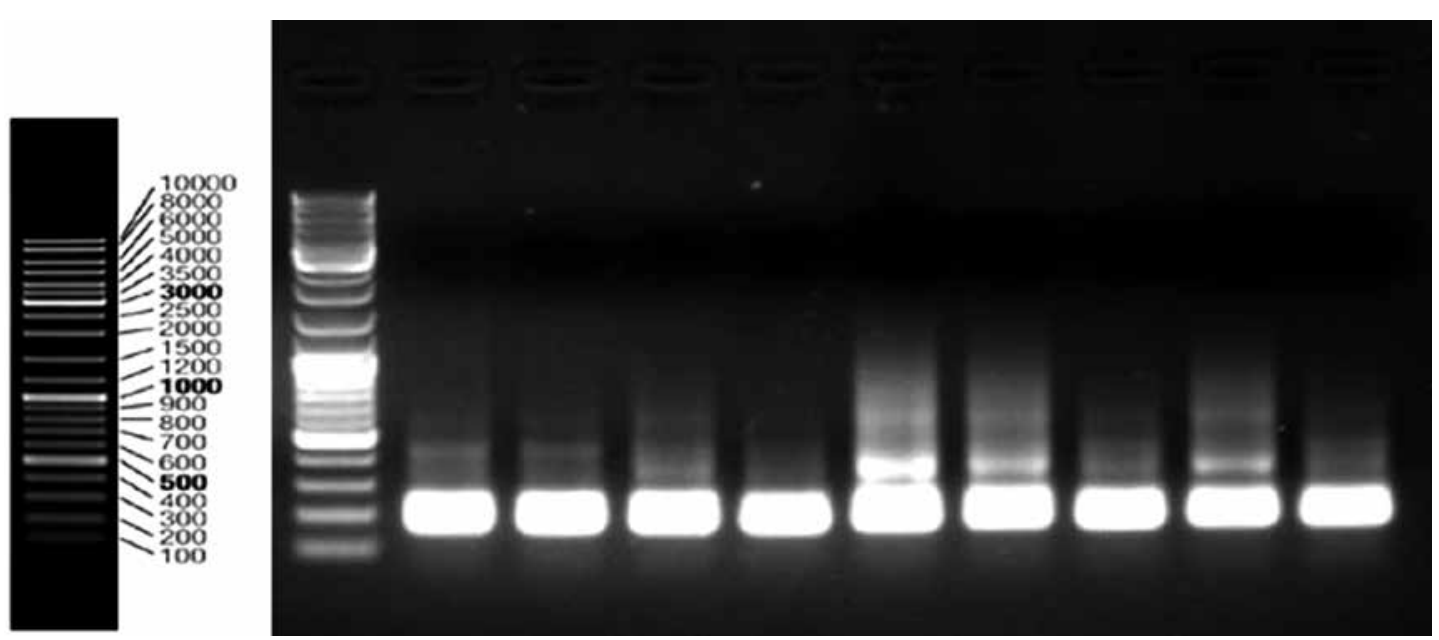

Figure 4. DGGE profile of EPS obtained from subjects with BPH (A2) and prostate cancer (B-2)

sequence of the band examined showed about $97 \%$ similarity. Among these strains, the one that showed the highest similarity of the same category was selected. Based on these results, the phylogenetic tree was created by MEGA 5.05 (Figure 5).

As shown in the phylogenetic tree (Figure 5), most of the strains were uncultured and values

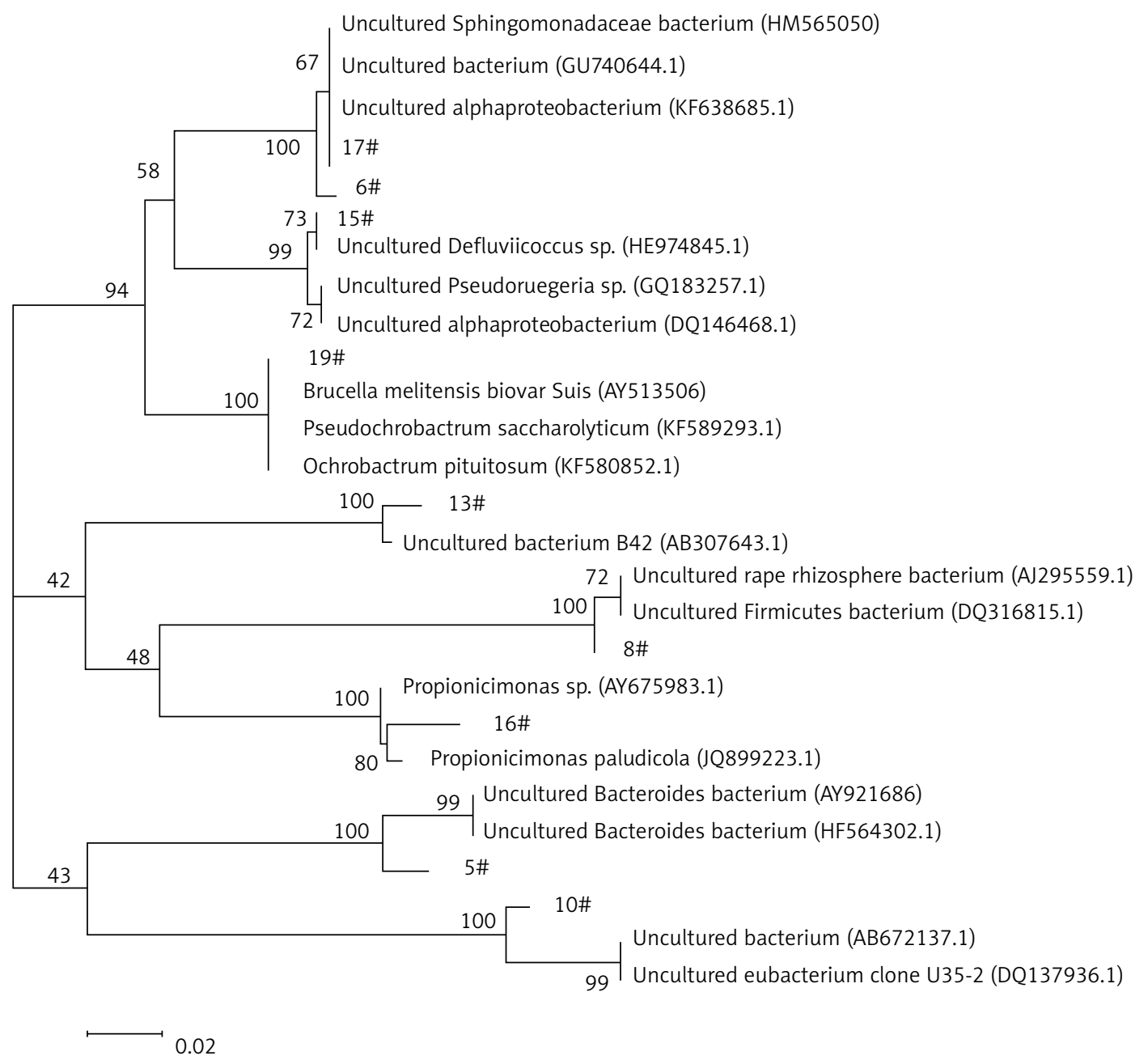

Figure 5. Phylogenetic tree of distinguished strains in prostate cancer group and BPH group 


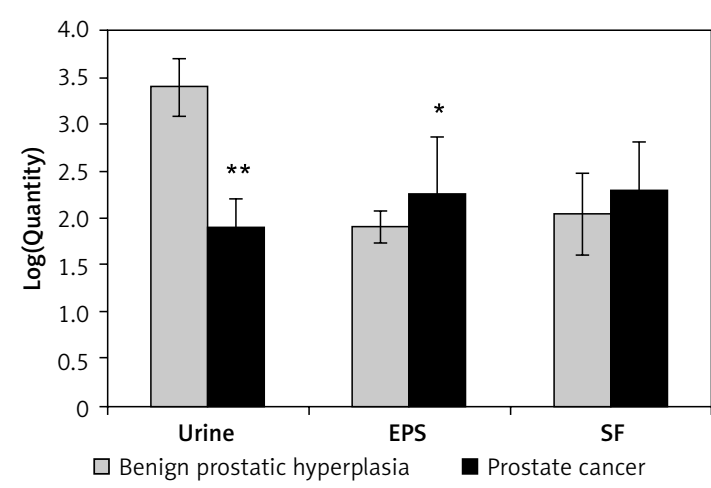

Figure 6. The qPCR results of $E$. coli in urine, EPS and seminal fluid (SF) obtained from subjects with $\mathrm{BPH}$ and prostate cancer

${ }^{*} p<0.05,{ }^{* *} p<0.01$; based on Students' t test.

on the branches of the tree showed a high confidence coefficient and close genetic relationships.

\section{Quantitative polymerase chain reaction assays}

The standard curves of both two PCR assays (E. coli and Enterococcus) showed a good linearity. The amount of $E$. coli in the prostate cancer group was statistically significantly decreased in urine and increased in EPS $(p<0.05)$, but showed no significant change in seminal fluid compared with the BPH group (Figure 6). The amount of Enterococcus in EPS in the prostate cancer group was significantly higher $(p<0.05)$ than that seen in the $\mathrm{BPH}$ group, while the amount of Enterococcus in urine of the prostate cancer group was significantly lower $(p<0.05)$ compared to that noted in the $\mathrm{BPH}$ group (Figure 7 ). In the seminal fluid, the quantity of Enterococcus in the prostate cancer group was found to be significantly higher $(p<0.01)$ compared to the BPH group (Figure 7).

\section{Discussion}

Previously, it was reported that prostatitis and prostatitis symptoms are associated with an increased risk of prostate cancer [5]. It is likely that bacteria might induce a chronic inflammatory state in the prostate that results in enhanced production of pro-inflammatory cytokines. Both neutrophils and macrophages may release proinflammatory molecules such as nitric oxide that have the propensity to cause genetic damage that could pave the way for enhanced cell proliferation and cancer. This is particularly interesting in the light of the reports that low-grade inflammation exists in cancer. Some studies did show that specific types of bacterial sequences could be present in prostatic tissue that may not be detected by traditional cell culture methods [20].

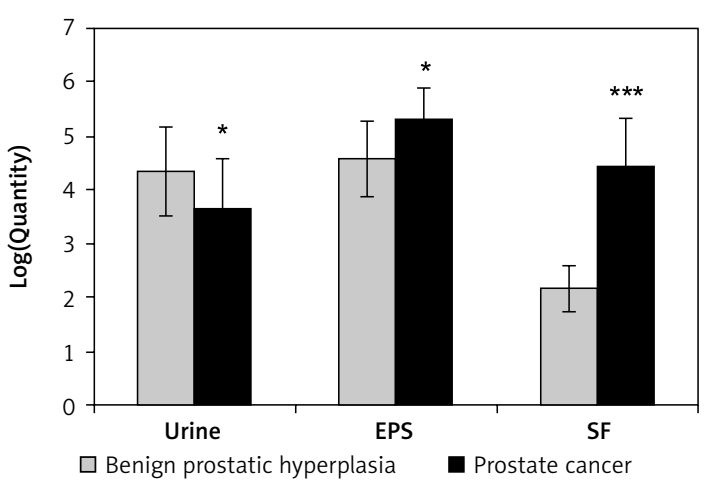

Figure 7. The $\mathrm{qPCR}$ results of Enterococcus in urine, EPS and seminal fluid (SF) obtained from subjects with $\mathrm{BPH}$ and prostate cancer

${ }^{*} p<0.05,{ }^{* * *} p<0.01$; based on Student's t test.

Molecular-based methods to identify and characterize microorganisms have contributed to microbial discovery. 16S rDNA-based PCR assays are more sensitive than the traditional methods that rely on microbial culture techniques [21, 22]. Some previous studies evaluated bacterial 16S rDNA sequences in prostatic tissue from patients with prostate cancer [23]. In the present study, using a combination of PCR-DGGE fingerprinting analysis and sequencing, combined with phylogenetic analysis, we observed that the microbiota present in the urinary system are significantly different between patients with $\mathrm{BPH}$ and prostate cancer.

Denaturing gradient gel electrophoresis fingerprints indicated that the urinary microbiota compositions in the EPS of prostate cancer were similar to those seen in $\mathrm{BPH}$ though there were significant differences in the quantities of specific strains between the groups. The sequencing results showed that EPS of prostate cancer patients were rich in Bacteroidetes bacteria, Alphaproteobacteria, Firmicutes bacteria, Lachnospiraceae, Propionicimonas, Sphingomonas, and Ochrobactrum, which might be involved in the progression of prostate cancer. Capsular polysaccharides produced by Bacteroidetes bacteria are important pathogenic factors that can cause suppurative lesions in the abdominal cavity and other organs. Sphingomonas is a genus of Gram-negative bacteria that belongs to the Alphaproteobacteria, which is one of the commonest groups associated with urinary tract infection. Hence, increase of Alphaproteobacteria (band \#6 and \#17) in the prostate cancer group may be relevant to the infection and inflammation of the prostate seen in patients with prostate cancer. Firmicutes bacteria account for the largest proportion of the human gut microbiome and are known to be involved in energy resorption and obesity [24]. The number of Firmicutes in vertebrates including humans is considered to be diet-dependent and correlates 
with caloric intake. Since a high-fat and high-caloric diet is widely believed to be an important factor involved in the genesis of prostate cancer, it is reasonable to propose that the increased number of Firmicutes (band \#8 and \#13) seen in the EPS of patients with prostate cancer may have a role in its pathogenesis. Propionicimonas belongs to the Propionibacterineae, which are the main anaerobic bacteria in EPS of prostatitis patients. Ochrobactrum is an opportunistic pathogen. In the present study, we observed that the quantity of Ochrobactrum in the EPS of prostate cancer patients was much higher compared with $\mathrm{BPH}$, which suggests the immune dysfunction of these patients. This implies that efforts to suppress the growth of Ochrobactrum could be of significant benefit in the prevention of prostate cancer development.

The results of the present investigation showed that $E$. coli and Enterococcus are present in significantly large number in the EPS and seminar fluid of subjects with prostate cancer compared to $\mathrm{BPH}$. Increased levels of these two types of bacteria in EPS and seminal fluid indicate that a significant degree of inflammation occurs in those with prostate cancer.

Several previous studies have used prostate cancer tissue to detect the presence and influence of bacteria in prostate cancer $[23,25,26]$. In contrast, we collected EPS to investigate the presence and possible shift in the type and quantity of microbiota in $\mathrm{BPH}$ and prostate cancer. Expressed prostatic secretions are secreted by the prostate and pass through the urethra, which is connected to the skin. Hence, it is likely that bacteria in the male reproductive tract and urethra could access EPS, and so their analysis could more likely reflect a shift in the type of bacteria present in the prostate with different diseases.

In conclusion, bacterial flora in EPS of subjects with prostate cancer is different from that seen in $\mathrm{BPH}$. These results suggest that ecological dysbiosis of the bacterial community in the EPS might play an important role in the pathobiology of prostate cancer.

\section{Conflict of interest}

The authors declare no conflict of interest.

\section{References}

1. Palapattu GS, Sutcliffe S, Bastian PJ, et al. Prostate carcinogenesis and inflammation: emerging insights. Carcinogenesis 2005; 26: 1170-81.

2. Piastowska-Ciesielska AW, Kozlowski M, Wagner W, et al. Effect of an angiotensin II type 1 receptor blocker on caveolin-1 expression in prostate cancer cells. Arch Med Sci 2013; 9: 739-44.

3. Lax AJ, Thomas W. How bacteria could cause cancer: one step at a time. Trends Microbiol 2002; 10: 293-9.
4. Krieger JN, Riley DE, Cheah PY, et al. Epidemiology of prostatitis: new evidence for a world-wide problem. World J Urol 2003; 21: 70-4.

5. Cheng I, Witte JS, Jacobsen SJ, et al. Prostatitis, sexually transmitted diseases, and prostate cancer: the California Men's Health Study. PLoS One 2010; 5: 8736.

6. Vartoukian SR, Palmer RM, Wade WG. Strategies for culture of 'unculturable' bacteria. FEMS Microbiol Lett 2010; 309: 1-7.

7. Handschur M, Pinar G, Gallist B, et al. Culture free DGGE and cloning based monitoring of changes in bacterial communities of salad due to processing. Food Chem Toxicol 2005; 43: 1595-605.

8. Muyzer G, de Waal EC, Uitterlinden AG. Profiling of complex microbial populations by denaturing gradient gel electrophoresis analysis of polymerase chain reaction-amplified genes coding for $16 \mathrm{~S}$ rRNA. Appl Environ Microbiol 1993; 59: 695-700.

9. Hou DS, Long WM, Shen J, et al. Characterisation of the bacterial community in expressed prostatic secretions from patients with chronic prostatitis/chronic pelvic pain syndrome and infertile men: a preliminary investigation. Asian J Androl 2012; 14: 566-73.

10. Jiang W, Jiang Y, Li C, et al. Investigation of supragingival plaque microbiota in different caries status of Chinese preschool children by denaturing gradient gel electrophoresis. Microb Ecol 2010; 61: 342-52.

11. Ling Z, Kong J, Liu F, et al. Molecular analysis of the diversity of vaginal microbiota associated with bacterial vaginosis. BMC Genomics 2010; 11: 488.

12. Allaker RP. Investigations into the micro-ecology of oral malodour in man and companion animals. J Breath Res 2010; 4: 1-6.

13. Riemersma WA, van der Schee CJC, van der Meijden WI, et al. Microbial population diversity in the urethras of healthy males and males suffering from non-chlamydial, nongonococcal urethritis. J Clin Microbiol 2003; 41: 1977-86.

14. Wei H, Dong L, Wang T, et al. Structural shifts of gut microbiota as surrogate endpoints for monitoring host health changes induced by carcinogen exposure. FEMS Microbiol Ecol 2010; 73: 577-86.

15. Soares DM, Figueiredo MJ, Martins JM, et al. A crucial role for IL- 6 in the CNS of rats during fever induced by the injection of live E. coli. Med Microbiol Immunol 2012; 201: 47-60

16. Zwielehner J, Liszt K, Handschur M, et al. Combined PCR-DGGE fingerprinting and quantitative-PCR indicates shifts in fecal population sizes and diversity of Bacteroides, bifidobacteria and Clostridium cluster IV in institutionalized elderly. Exp Gerontol 2009; 44: 440-6.

17. Pathak S, Awuh JA, Leversen NA, et al. Counting Mycobacteria in infected human cells and mouse tissue: a comparison between qPCR and CFU. PLoS ONE 2012; 7: 1-11.

18. Kline EE, Treat ED, Averna TA, et al. Citrate concentrations in human seminal fluid and expressed prostatic fluid determined via1H nuclear magnetic resonance spectroscopy outperform prostate specific antigen in prostate cancer detection. J Urol 2006; 176: 2274-9.

19. Chao SH, Tomii Y, Watanabe K, et al. Diversity of lactic acid bacteria in fermented brines used to make stinky tofu. Int J Food Microbiol 2008; 123: 134-41.

20. Riley DE, Berger RE, Miner DC, et al. Diverse and related 16S rRNA-encoding DNA sequences in prostate tissues of men with chronic prostatitis. J Clin Microbiol 1998; 36: 1646-52. 
21. Clarridge JE. Impact of $16 \mathrm{~S}$ rRNA gene sequence analysis for identification of bacteria on clinical microbiology and infectious diseases. Clin Microbiol Rev 2004; 17: 840-62.

22. Harris KA, Hartley JC. Development of broad-range $16 \mathrm{~S}$ rDNA PCR for use in the routine diagnostic clinical microbiology service. J Med Microbiol 2003; 52: 685-91.

23. Leskinen MJ, Rantakokko-Jalava K, Manninen R, et al. Negative bacterial polymerase chain reaction (PCR) findings in prostate tissue from patients with symptoms of chronic pelvic pain syndrome (CPPS) and localized prostate cancer. Prostate 2003; 55: 105-10.

24. Ley RE, Peterson, DA, Gordon JI. Ecological and evolutionary forces shaping microbial diversity in the human intestine. Cell 2006; 24: 837-48.

25. Alexeyev O, Bergh J, Marklund I, et al. Association between the presence of bacterial 16S RNA in prostate specimens taken during transurethral resection of prostate and subsequent risk of prostate cancer (Sweden). Cancer Causes Control 2006; 17: 1127-33.

26. Sfanos KS, Sauvageot J, Fedor HL, et al. A molecular analysis of prokaryotic and viral DNA sequences in prostate tissue from patients with prostate cancer indicates the presence of multiple and diverse microorganisms. Prostate 2008; 68: 306-20. 